\title{
The Diffusion-Circulation Coupling in Stellar Radiative Zones: 2D Simulation
}

\author{
Sylvie Théado and Sylvie Vauclair \\ Observatoire Midi-Pyrénées, Laboratoire d'Astrophysique, 14 Avenue \\ Edouard Belin, 31400 Toulouse, France
}

\begin{abstract}
We have done a $2 \mathrm{D}$ simulation of meridional circulation in the presence of helium diffusion below stellar convective zones. For slowly rotating stars the microscopic diffusion of helium leads to a $\mu$-gradient which has an important effect on the rotation induced mixing. We present here the results of this $2 \mathrm{D}$ simulation for solar type stars.
\end{abstract}

\section{Introduction}

In case of small differential rotation, the radial and horizontal components of the meridional velocity can be written :

$$
u_{r}=U_{r} P_{2}(\cos \theta) \quad u_{\theta}=-\frac{1}{2 \rho r} \frac{d}{d r}\left(\rho r^{2} U_{r}\right) \sin \theta \cos \theta
$$

where :

$$
U_{r}=\frac{P}{\rho g T C_{p}\left(\nabla_{a d}-\nabla+\nabla_{\mu}\right)} \frac{L}{M_{*}}\left(E_{\Omega}+E_{\mu}\right)
$$

$U_{r}$ is the sum of two terms : the classical Eddington-Sweet term $E_{\Omega}$ and the $\mu$-gradients term $E_{\mu}\left(\Lambda\right.$ refers to the horizontal $\mu$-fluctuations $\left.\frac{\tilde{\mu}}{\bar{\mu}}\right)$ :

$$
\begin{aligned}
& E_{\Omega}=\frac{8}{3}\left(\frac{\Omega^{2} r^{3}}{G M}\right)\left(1-\frac{\Omega^{2}}{2 \pi G \bar{\rho}}\right) \\
& E_{\mu}=\frac{\rho_{m}}{\bar{\rho}}\left\{\frac{r}{3} \frac{d}{d r}\left[\left(H_{T} \frac{d \Lambda}{d r}\right)-\left(\chi_{\mu}+\chi_{T}+1\right) \Lambda\right]-\frac{2 H_{T} \Lambda}{r}\right\}
\end{aligned}
$$

In the most general case, $\mu$-currents are opposite to $\Omega$-currents. In order to compute in a more realistic way the coupling between helium settling and the two meridional currents we have performed a $2 \mathrm{D}$ numerical simulation which computes the helium abundance variations under the effects of helium diffusion and meridional circulation. No free parameter is introduced in these computations, although some simplifying assumptions are made (static model, constant rotation velocity). As an initial model we use a standard homogeneous $0.75 \mathrm{M}_{\odot}$ halo star model. A complete description of the numerical method and the equations introduced in the simulation can be found in Théado \& Vauclair (2003a) while the process is analytically described in Vauclair \& Théado (2003). 


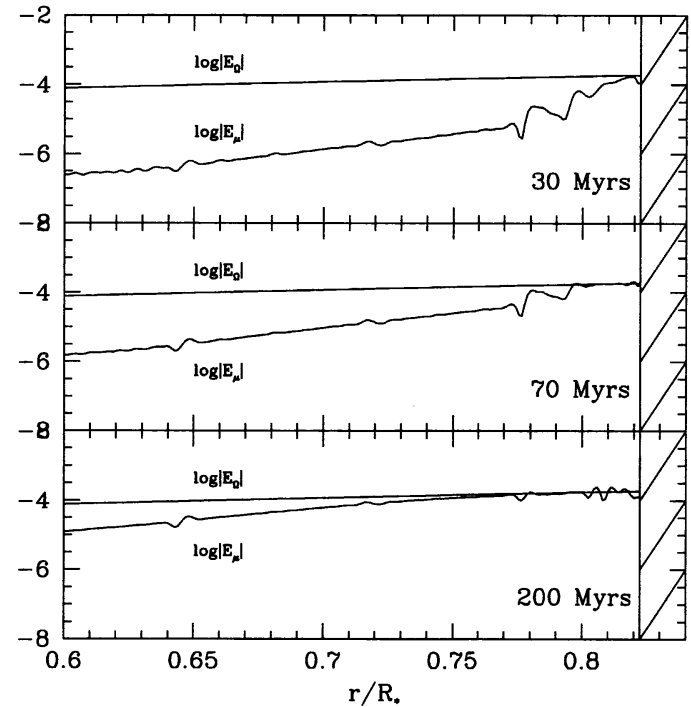

Figure 1. Simulation including diffusion, $\Omega$-currents and $\mu$-currents: $E_{\Omega}$ and $E_{\mu}$ profiles below the convective zone at different steps of the simulation.

\section{Computational Results}

Figure 1 displays the $\left|E_{\Omega}\right|$ and $\left|E_{\mu}\right|$ profiles inside the stars at different steps of the simulation. The $\mu$-currents become rapidly of the same order of magnitude as the $\Omega$-currents below the convective zone. As soon as the two currents compensate each other, the meridional circulation seems to freeze out in the concerned regions. However when the circulation vanishes, the helium settling proceeds which may alter the balance between the two opposite currents.

In Théado and Vauclair (2003a), we show that helium falling from the mixed outer regions decreases $\mu$-gradients below the convective zone. It breaks the equilibrium between $\left|E_{\Omega}\right|$ and $\left|E_{\mu}\right|$ and triggers the circulation again: a new circulation loop develops which mixes up into the convective zone the region polluated by diffusion. It acts as a restoring system for the $\left|E_{\Omega}\right|-\left|E_{\mu}\right|$ balance with a time scale much smaller than the diffusion one, which explains why the $E_{\Omega}$ and $E_{\mu}$ profiles remain very close on figure 1.

This suggests that diffusion and mixing react in such a way so as to keep both the horizontal and vertical $\mu$-gradients constant in the frozen region thereby increasing in a significant way the settling time scale. Such a process has been applied to the lithium problem in halo stars (Théado \& Vauclair 2001) and to the lithium dip in the Hyades (Théado \& Vauclair 2003b)

\section{References}

Théado, S., Vauclair, S., 2001, A\&A 375, 70-86

Théado, S., Vauclair, S., 2003a, ApJ in press, astro-ph/0301246

Théado, S., Vauclair, S., 2003b, ApJ in press, astro-ph/0301203

Vauclair, S., Théado, S., 2003, ApJ in press, astro-ph/0301202 